\section{$\triangle 151$}

LEUKOTRIENES IN PROTEIN ENERGY MALNUTRITION: NEW ASPECTS IN THE PATHOPHYSIOLOGY OF KWASHIORKOR. Ertan Mayatepek, Katja Becker, Georg F. Hoffmann, Michael Leichsenring. University Children's Hospital, Heidelberg, Germany The actions of cysteinyl leukotrienes include production of oedema. We investigated whether these mediators might be involved in the oedematous malnutrition syndrome kwashiorkor. Methods: The capacity of leukotriene (LT) synthesis by stimulated whole blood and urinary LTE 4 excretion was measured by specific immunoassays after HPLC purification in 12 children with kwashiorkor, and compared with that in 24 marasmic and 12 control children. Results: Urinary LTE 4 excretion was significantly higher in patients with kwashiorkor when compared to controls $(118.8 \pm 28.5$ vs $31.1 \pm 19.3 \mathrm{nmol} / \mathrm{mol}$ creatinine, $\mathrm{p}<0.01$ ). Whole blood cysteinyl LT synthesis was increased in kwashiorkor patients by a factor of $3.5(p<0.01)$. In marasmic children, LTE 4 excretion and whole blood cysteinyl LT synthesis did not differ from those in controls. LTB 4 synthesis, however, was greatly reduced in kwashiorkor patients $(11.5 \pm 2.4$ vs $46.5 \pm 6.4 \mathrm{ng} / \mathrm{mL}$ $p<0.01$ ). Conclusion: Inability to synthesise the immunoregulator LTB4 may lead to inefficient chemoattraction of phagocytes and an inadequate inflammatory response in kwashiorkor. The increased endogenous cysteinyl LT generation in kwashiorkor suggests that these lipid mediators are involved in the pathophysiology of the syndrome, esp. in oedema formation.
152

NNCREASED URINARY EXCRETION OF DICARBOXYLIC ACIDS AND 4-HYDROXY PHENYLLACTIC ACID IN PATIENTS WITH ZELLWEGER \$YNDROME Ertan Mayatepek, Claudia K. Seppel, Georg F. Hoffmann. University Children's Hospital, Heidelberg, Germany

B-Oxidation of dicarboxylic acids has been shown to occur in mitochondria and peroxisomes of rat liver in vitro. Patients with Zellweger syndrome (ZS) offer a unique possibility to study the importance of peroxisomes in the degradation of dicarboxylic acids in vivo. Methods: We quantified urinary organic acids in seven ZS patients with impaired peroxisomal B-oxidation but normal mitochondrial B-oxidation capacity by GCMS. Results: Increased amounts of dicarboxylic acids with an odd number of carbon atoms (e.g. azelaic (up to $23 \mathrm{vs}<0.1 \mathrm{mmol} / \mathrm{mol}$ creatinine in controls), pimelic acid (up to $15 \mathrm{vs}<0.1$ )) or an even number of carbon atoms (e.g. adipic (up to $180 \mathrm{vs}<0.1$ $\mathrm{mmol} / \mathrm{mol}$ creatinine in controls), suberic (up to $140 \mathrm{vs}<0.1$ ), sebacic acid (up to 200 vs $<0.1)$ ) and 2 -hydroxysebacic acid, a medium-chain hydroxydicarboxylic acid (up to $50 \mathrm{vs}<0.1 \mathrm{mmol} / \mathrm{mol}$ creatinine in controls), were found in the urines of these patients. In addition, patients with ZS showed an hiohly elevated excretion of 4-hydroxyphenyllactic acid (up to 98 vs $<0.1 \mathrm{mmol} / \mathrm{mol}$ creatinine in controls). Conclusion: These results prove the functional importance of peroxisomes in the $B$-oxidation of these compounds in vivo. The characteristic pattern of urinary organic acids in ZS should prompt for specific diagnostic procedures for peroxisomal disorders, when found in patients undergoing selective screening for inherited metabolic diseases.

\section{$\triangle 154$}

EFFECT OF STRUCTURED ABSTRACTS (SA) ON ABSTRACT QUALITY Neit Mclntosh", Gabriel Duc ${ }^{\wedge}$ and Gunnar Sedin "- "Universities of Edinburgh, UK; ^Zurich, Switzerland; and "Uppsala, Sweden Hypothesis: SA contain more information than unstructured. Study design: A comparison of original abstracts $(O A)$ and resubmitted SA. Setting: Pairs of OA and resubmitted SA to the ESPR, 1993

"Subjects": Following receipt of $O A$, authors were asked to resubmit using structured format suggested by the Working Group on Critical Appraisal of Medical Literature, Ann Intern Med. 106:598-604. 72 were resubmitted on the same abstract form (SA form), 94 on one side of A4 (SA A4) - as requested. Measurements: Each reviewer (2 ex presidents ESPR) assessed 1 abstract from each pair giving a Scientific score out of 6 and a time for review. The information content (IC) was then objectively measured by third ex president. Results:
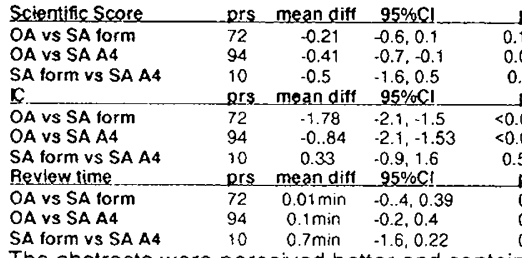
0 28 paired t test Conclusion: The abstracts were perceived better and contained more information when submitted in a structured format. The IC can be increased very significantly without necessarily increasing abstract size or review time.
A RANDOMISED TRIAL OF COMPUTERISED TREND MONITORING Neil Mclntosh. Steve Cunningham and Rob Elton*. Departments of Child Life and Health and Medical Statistics*, University of Edinburgh.

Hypothesis: Neonatal computerised trend monitoring (CTM) reduces the morbidity and mortality of infants receiving intensive care.

Study type/Setting: Prospective cohort study in a tertiary neonatal service. Patients/Interventions: 445 consecutive newborn who required neonatal intensive care were randomised to receive CTM in addition to the use of conventional monitors (CM) or just CM. The CTM provides a graphical trend over time periods varying from 6 mins to 3 days for any combination (to a maximum of 5) of the monitored physiological or environmental parameters. Measurements: The following short term measures were compared daily ove the first 7 days of life - periventricular haemorrhage (with grade - $G$ (out of 4 ) flares - F (+l-), cysts - C (+l-) and ventricular size-VS (mms); doppler of the anterior cerebral artery - Rl; volume of colloid support - $\mathrm{Vol}(\mathrm{m} / \mathrm{s})$, and number of blood gases performed - BG (n). The outcome measures were death - D, abnormal ultrasound - US, percent discharged home $D / C$, time to death/discharge - tD/C (d), time ventilated - $\mathrm{tV}(\mathrm{d})$ and time in oxygen - tO2(d). Results: G F\% C\% VS RI VOl BG D\% US\% D/C tD/C IV 102 $\begin{array}{llllllllllllll}\text { CTM } & 0.27 & 17 & 2 & 10.3 & 0.74 & 7.1 & 3.6 & 11 & 24 & 53 & 19 & 2 & 3 \\ \mathrm{CM} & 0.23 & 15 & 3 & 10.5 & 0.74 & 7.2 & 3.5 & 9 & 27 & 60 & 21 & 2 & 3\end{array}$ $\begin{array}{llllllllllllll}C M & 0.23 & 15 & 3 & 10.5 & 0.74 & 7.2 & 3.5 & 9 & 27 & 60 & 21 & 2 & 3\end{array}$ for quantitative outcomes and unpaired t test for short term measures - all NS Conclusions: CTM introduced into the management of intensive care neonates could not be demonstrated to improve short term outcome.

\section{$\Delta 153$}

TRACHEAL IL8 AS A PREDICTOR OF CHRONIC LUNG DISEASE Jan McColm, Neil Mclntosh

Department of Child Life and Health, University of Edinburgh.

Hypothesis: Is the neutrophil activating cytokine 1 L8 associated with chronic inflammation and development of chronic lung disease in the immature infant? Study type/Setting: Prospective cohort study in a tertiary neonatal service. Patients: 12 consecutive newborn $=<30$ weeks gestation (median 27). Interventions: 8 infants received surfactant because of respiratory distress syndrome (rescue situation) and all were ventilated using a standard technique, Measurements: tracheal secretions and washings were collected daily for 7 days at the time of normal endotracheal suction. The IL 8 concentrations of the supernatants were measured by a specific RIA (cv 5,13\%). Chronic Lung Disease was defined as an oxygen requirement at 28 days of age. Results: 7 of the 12 infants ( $58 \%$ ) developed chronic lung disease typical of bronchopulmonary dysplasia - 2 did not receive surfactant as they initially did not have an oxygen requirement or a diagnosis of RDS. 5 infants $(42 \%)$ did not develop chronic lung disease - 3 received surfactant because of the initial diagnosis of RDS. All infants survived. The median (range) of 1 L 8 in the tracheal secretions on day 2 in the group who did not develop BPD was $5.7(0.4-20.7) \mathrm{mcg} / \mathrm{l}$ : the corresponding levels in the group going on to develop CLD was $42(22-352) \mathrm{mcg} / \mathrm{ml}(\mathrm{p}=0.042$, Mann-Whitney $U$ test $)$

Conclusions: The acute inflammatory cytokine $\mathrm{L} 8$, is present at high

concentrations early in the tracheal secretions of babies who go on to develop chronic lung disease.

\section{$\triangle 156$}

\section{ABSTRACT ASSESSMENT - HOW OBJECTIVE CAN IT BE?} Mclntosh $\mathrm{N}$ - Dept of Child Life and Health, University of Edinburgh.

Introduction: The abstract is the window for the reader to assess the work Hypothesis tested: Specialists and non specialists in a scientific field should assess abstracts similarly if the abstract is well prepared.

Study design: A comparative study by members of a society council $(n=9)$ and subspecialist working groups (epidemiology, $n=5$ : neonatology, $n=6$ ).

Setting: Abstracts submitted to the ESPR, 1993.

"Subjects": 14 epidemiology abstracts and 111 neonatal abstracts.

Measurements: Instructions to the council and subspecialist working group members were the same - "Grade abstract for quality out of $6(1$, very poor; 2 , poor; 3 , adequate; 4 , good; 5 , very good; 6 , excellent)". Comparison (mean, SD) was by paired t test and correlation. The SD around the mean indicated uncertainty about the "quality" of the work.

Results:

Epidemiology abstracts $(n=14)$

difference $r \quad 95 \% \mathrm{Cl} \quad \mathrm{p}$ working group $\vee$ council (mean) working group $v$ council (SD) correlation (mean marks)

Neonatology abstracts $(n=111)$ working group $v$ council (mean) working group $v$ council (mean)
corking group $v$ council (SD)
correlation (mean marks)

$$
\begin{array}{cccc}
0.61 & & 0.331 ; 0.92 & 0.0007 \\
0.07 & & -0.16: 0.30 & 0.52 \\
& 0.75 & 0.39 ; 0.91 & 0.0012 \\
& & & \\
0.22 & & 0.14: 0.30 & <0.0001 \\
-0.07 & & -0.14 ; 0.01 & 0.079 \\
& 0.74 & 0.64: 0.81 & <0.0001
\end{array}
$$

Conclusion: Although there was a highly significant co in the field perceive work as significantly less good/important. This judgement does not appear to be related to uncertainty about the quality of the work. 